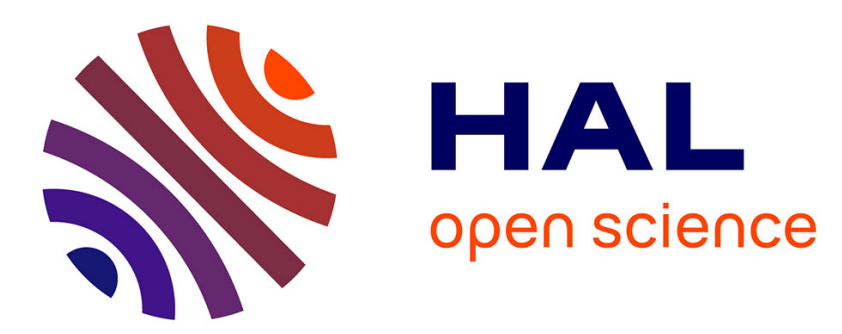

\title{
Code Domain Non Orthogonal Multiple Access versus ALOHA: a simulation based study
}

Diane Duchemin, Jean-Marie Gorce, Claire Goursaud

\section{To cite this version:}

Diane Duchemin, Jean-Marie Gorce, Claire Goursaud. Code Domain Non Orthogonal Multiple Access versus ALOHA: a simulation based study. ICT 2018 - 25th International Conference on Telecommunications, Jun 2018, Saint-Malo, France. pp.445-450, 10.1109/ICT.2018.8464836 . hal-01801103

\section{HAL Id: hal-01801103 \\ https://hal.inria.fr/hal-01801103}

Submitted on 28 May 2018

HAL is a multi-disciplinary open access archive for the deposit and dissemination of scientific research documents, whether they are published or not. The documents may come from teaching and research institutions in France or abroad, or from public or private research centers.
L'archive ouverte pluridisciplinaire HAL, est destinée au dépôt et à la diffusion de documents scientifiques de niveau recherche, publiés ou non, émanant des établissements d'enseignement et de recherche français ou étrangers, des laboratoires publics ou privés. 


\title{
Code Domain Non Orthogonal Multiple Access versus AlOHA: a simulation based study
}

\author{
Diane Duchemin, Jean-Marie Gorce, Claire Goursaud \\ CITI laboratory \\ Univ Lyon, Inria, INSA Lyon \\ Lyon, France \\ surname.name@insa-lyon.fr
}

\begin{abstract}
Non Orthogonal Multiple Access (NOMA) is expected to play an important role for IoT networks, allowing to reduce signaling overheads and to maximize the capacity of dense networks with multiple packets simultaneous transmission. In the uplink, NOMA can improve significantly the performance of an ALOHA random access if the receiver implements a multi-user detection algorithm. In this paper we compare the performance of a code domain NOMA with a classical ALOHA protocol, through simulations. The code domain NOMA uses random Gaussian codes at the transmitters and exploits compressive sensing at the receiver to maximize users detection and to minimize symbol error rates.
\end{abstract}

Index Terms-Internet of Things, Random access, Non Orthogonal Multiple Access, Compressive sensing, ALOHA.

\section{INTRODUCTION}

The context of this study is the transmission of short and independent information quantities from a dense population of nodes to a single base station (BS) under high reliability, low latency and low power consumption constraints. This transmission scenario is crucial for massive Internet of Things (IoT) Low Power Wide Area Network (LPWAN) [1] with Ultra Reliable and Low Latency Communications (URLLC) constraints [2].

In order to provide the nodes with quasi-instantaneous and on-demand communications, the hand-shake protocol has to be minimal and a direct random network access without primary connection is seducing. Indeed, on account of latency constraint and energy efficiency, high overhead and delay induced in classic resource allocation protocols prevent from using a connection-oriented mode in this context. This problem is one of the main target of the future 5G NB-IoT protocol, but the investigated solutions are based on a three-step handshake mechanism at the minimum (instead of five in usual $4 \mathrm{G}$ or $5 \mathrm{G}$ protocols).

The most classical protocol-free multiple access scheme is AlOHA [3] but it suffers from poor performance when the number of transmitters and the transmission probability grow simultaneously due to a high collision probability. At the protocol level, the collision probability can be kept low by

This work has been partly supported by the INSA Lyon - SPIE chair on the Internet of Things and by the French National Agency for Research (ANR) under grant $\mathrm{n}^{\circ}$ ANR-16-CE25-0002 - EPHYL increasing the frequency bandwidth allocated to the random access channel and multi-slotted ALOHA techniques, extensively studied in the past [4], can be chosen.

An alternative, investigated at the Physical layer relies on the use of Non-Orthogonal Multiple Access (NOMA) techniques associated with a powerful multi-user detection algorithm at the receiver [5]. NOMA techniques can be designed in the power, code or time-frequency domains. Power-domain NOMA relies on a classic simultaneous multiple access (MA) using a Superposition Coding (SC) strategy in order to allow more users to access the same resource. On the receiver side, Successive Interference Cancellation (SIC) is employed for recovering each communication. A perfect recovery is possible only if the transmission powers or the individual rates are tuned as a function of the channel states. Another category consists in multiplexing the competing users in the code domain by using user-specific spreading sequences, having a sparse, low-density and low inter-correlation properties. One of the advantages of such MA techniques is the grant-free access provided by the non-orthogonality feature, but at the cost of introducing users interference. This kind of technique can be seen as a step back to the Code Division Multiple Access (CDMA) technology extensively studied in the 90 s. The most important difference relies in the fact that in the context of IoT, the receiver doesn't know in advance the active codes and has to detect them and to estimate their data. The recent contribution in this field relies on the use of the sparsity assumption allowing to use algorithms based on compressive sensing (CS) [6]-[11]. Last but not least, we should mention that another popularized technique is the use of SCMA [12] which redefines modulation and code multiplexing as a whole.

In this paper, we focus on the Code domain NOMA following [9]-[11], where Gaussian random codes and CS are used. Following Xie et al. [11] we use their proposed algorithm for the decoding task.

Our work compares the performance of such approach with the performance achievable with a standard ALOHA technique, taken as a reference in terms of uncoordinated random access scheme. As Choi in [13], we show that NOMA allow a performance improvement compared to a slotted ALOHA access. While [13] is dedicated to Power domain NOMA with an analysis of the throughput in multichannel ALOHA, our study 
deals with Code domain NOMA in a single channel ALOHA scheme. Based on the implementation of the proposition of [11], we evaluate the gains of NOMA with different metrics, covering both the users detection and the data estimation tasks.

The rest of the paper is organised as follows: section II describes our model, with the two confronted access types. Section III details the metrics used for the performance comparison. A theoretical analysis of detection performance in a simple scenario is realized in section IV while the actual detection and estimation are jointly retrieved and evaluated in section V under a more realistic scenario. Section VI concludes the paper.

\section{MODEL DESCRIPTION}

\section{A. Scenario}

The scenario herein proposed follows [11]. The reference topology is a single cell in the uplink, with a base station (BS) made of a unique receiver equipped with multiple antennas. A very dense population of users (herein called nodes) is spread over the cell and generates random and bursty transmissions. The nodes set is referred to as $\mathcal{N}$, whose size is $N=\operatorname{card}(\mathcal{N})$.

The BS emits a synchronization frame periodically, allowing the nodes to work in a slotted manner. It is assumed that an unknown and small subset of nodes $\mathcal{N}_{a} \subset \mathcal{N}$ is active simultaneously in a given time slot. Their number is noted $N_{a}$. Thus the medium is a single resource, shared among users.

The wireless channel is modeled as follows. The pathloss is assumed to be known by the nodes which use power control to compensate it for. Therefore, the BS then receives all packets at the same power in average, but due to block fading the different signals are received at the BS with different powers. More precisely, the channel between each node and the BS, equipped with $\alpha$ antennas, is characterized by identically and independently distributed (i.i.d.) random variables. Note that all channel coefficients remain constant in a time slot. An Additive White Gaussian Noise (AWGN) is also considered.

The signal received at the BS can be expressed as follows:

$$
Y=\sqrt{\rho_{0}} \sum_{n=1}^{N_{a}} \mathbf{h}_{\mathbf{n}} \mathbf{x}_{\mathbf{n}}^{T}+Z
$$

where $Y \in \mathbf{C}^{\alpha \times m}$ is the received signal, $\rho_{0}$ is the Signal to Noise Ratio (SNR). The vector $\mathbf{h}_{\mathbf{n}}$ denotes the channel response of node $n$, whose each of the $\alpha$ elements is the fading coefficient for one of the BS antennas during the $m$ channel uses of the message $\mathbf{x}_{\mathbf{n}} . Z$ denotes the AWGN. [.] $]^{T}$ is the transpose operator. All elements of $Z$ and $\mathbf{h}_{\mathbf{n}}$ follow a standard complex normal law. The specific expressions of $\mathbf{x}_{\mathbf{n}}$ for each multiple access type are given hereunder.

\section{B. Aloha-type system}

1) Slotted AlOHA transmissions scheme: For a fair comparison, and as the assumptions in II-A hold for both NOMA and AlOHA transmissions, we consider a slotted ALOHA scheme.

Our ALOHA-based system does not use any coding strategy. The data stream is send as is, nevertheless, it is preambled by its node unique identifier $\mathbf{w}_{\mathbf{n}}$. A unique bit sequence is simply mapped to a node, the length $L$ of the identifier bit sequences is related to the nodes set size: $L=\log _{2}(N)$ bits. This sequence only requires a modulation before being preambled to the data: $\mathbf{w}_{\mathbf{n}}$ is the modulated symbols vector of the node $n$ identifier bit sequence. Let $\mathbf{s}_{\mathbf{n}} \in S^{d}$ denote the modulated symbols of data streams, where $S \subset \mathbb{C}$ is the set of modulated symbols. A message is thus obtained as follows:

$$
\mathbf{x}_{\mathbf{n}}=\left[\mathbf{w}_{\mathbf{n}}^{T} \mathbf{S}_{\mathbf{n}}^{T}\right]^{T}
$$

An active node, i.e. willing to send a message, will transmit on the next time slot whose length is $d+\lceil L / M\rceil$ where $\mathbf{M}$ is the chosen modulation's rate, and $\lceil$.$\rceil the ceiling function.$ Note that when the messages are dedicated to the nodes activity notification, only the identifiers are sent.

2) SIC decoder: The decoder of the slotted ALOHA-based transmission scheme exploits Successive Interference Cancellation (SIC). We assume that the decoder have prior information about the channel response for each node, their identifiers $\mathbf{w}_{\mathbf{n}}$ and the SNR. A predefined number of iterations $K$ is set. During each iteration, a node is detected before its data are estimated. The detection criteria is the lowest distance between the node's identifier sequence and its estimated symbols sequence (obtained with a hard decision on the result of a Least Square algorithm, given the channel response of the node, the SNR and the received signal $Y$ ). The data estimation is performed on the data part of the received signal with a Least Square (LS) algorithm as well, considering the SNR and the channel response of the detected node. The estimated contribution of the detected node is then canceled and another iteration starts.

\section{NOMA-type system}

The random code based approach proposed by Xie et al. in [11] is now described; the reader is refer to their paper for more details.

1) Code domain NOMA transmissions scheme: The use of Gaussian codes comes from Compressive Sensing (CS) theory. In CS theory, an initial signal of dimension $N$ is projected into a new signal composed of very few $(k)$ non-zero components among $N$, referred to as a $k$-sparse signal (or having a sparsity level $k$ ). The set of indices of these $k$ components constitutes the sparsity pattern. One then reconstructs this version of the signal from only $m$ measurements obtained by a specific $m * N$ measurement matrix with $m<N$. This under-determined problem can be solved thanks to an algorithm which performs a 11-minimization or a sparse approximation. The dimension's reduction of the observed signal (from $N$ to $m$ ) gives the compressive characteristic to this sensing technique. In [9], the authors establish the parallel between users detection and the sparsity pattern detection in CS, and refer to the On-Off Random Access channel for modeling a communication scheme where the signal sent by the $N$ users can be represented by a vector whose each entry corresponds to a user: if the user is active, its entry will be a non-zero value whereas an inactive user will be assigned a zero in the vector. The goal of the receiver is to detect the unknown active nodes rather than 
retrieving the actual value of the signal vector. Xie et al. [11] introduced a framework dedicated to simultaneous detectionestimation.

Similarly, each node $n$ is associated to a unique i.i.d. complex Gaussian coding normalized vector $c_{n}$ or column-normalized matrix $C_{n}$, which is used to encode the data $\mathbf{s}_{\mathbf{n}} \cdot \mathbf{x}_{\mathbf{n}} \in \mathbb{C}^{m}$ denotes an encoded message. For the general case:

$$
\mathbf{x}_{\mathbf{n}}=C_{n} \mathbf{s}_{\mathbf{n}},
$$

the received signal is then:

$$
Y=\sqrt{\rho_{0}} \sum_{n=1}^{N_{a}} \mathbf{h}_{\mathbf{n}}\left(C_{n} \mathbf{s}_{\mathbf{n}}\right)^{T}+Z .
$$

(For $d=1$, the equation 3 reduces to $\mathbf{x}_{\mathbf{n}}=\mathbf{c}_{\mathbf{n}} s_{n}$ and $s_{n}$ can be considered as an activity notification bit.)

2) NBOMP algorithm decoder: The Normalized Block Orthogonal Matching Pursuit is an iterative algorithm which, at each iteration, selects an additional potentially active node and jointly estimates the signals of previously selected nodes. Since it defines the number of detections, the iterations number $K$ should be at least as large as the expected number of active nodes to be able to detect all of them. The detection and estimation are respectively realized by means of correlation and Least Square (LS) operations and require the codes, SNR and channels knowledge. The word "block" in NBOMP refers to the block sparsity structure of the unknown signal and the block nodes signatures which appear when vectorizing equation 4 :

$$
\mathbf{y}=\sqrt{\rho_{0}} \sum_{n=1}^{N_{a}}\left(C_{n} \otimes \mathbf{h}_{\mathbf{n}}\right) \mathbf{s}_{\mathbf{n}}+\mathbf{z}=\sqrt{\rho_{0}} \sum_{n=1}^{N_{a}} B_{n} \mathbf{s}_{\mathbf{n}}+\mathbf{z}
$$

where $\operatorname{vec}(Y)=\mathbf{y}, \otimes$ denotes the Kronecker product, $B_{n}$ corresponds to the block signature matrix of node $n$.

For the detection task, at iteration $i$, a node $n$ is detected if its correlation coefficient $c_{n, i}$ is the largest of all remaining (undetected) nodes. $c_{n, i}$ is defined as follows:

$$
c_{n, i}=\frac{\left\|B_{n}^{H} * \mathbf{r e s}_{\mathbf{i}}\right\|^{2}}{\left\|\mathbf{h}_{\mathbf{n}}\right\|^{2}}
$$

where $[.]^{H}$ denotes the hermitian operator and res $_{\mathbf{i}}$ is the residual signal, i.e. the rest of the received signal $\mathbf{y}$ once the estimated symbols contributions (from the LS computation at iteration $i-1)$ are retrieved. When the $\max \left(c_{n, i}\right)$ is selected, the index $n$ is added to the set of detected nodes $\lambda_{i}$. The estimation is performed by a LS operation. Indeed, once the additional node is detected, the algorithm is intended to solve

$$
\tilde{\mathbf{s}}_{\lambda_{i}}=\underset{\mathbf{s}_{\mathbf{o}} \in S^{d . i}}{\arg \min }\left\|\mathbf{y}-\sqrt{\rho_{0}} B_{\lambda, i} \mathbf{s}_{\mathbf{0}}\right\|_{2}
$$

where $\tilde{s}_{\lambda_{\mathbf{i}}}$ is the concatenation of the estimated data streams of the $i$ detected nodes and $B_{\lambda, i}$ denotes the concatenation of the $i$ signature matrices $B_{n}$ with $n \in \lambda_{i}$. The MoorePenrose pseudo-inverse can be computed but, for the sake of complexity, our implementation of the NBOMP rather employs a QR factorization. A decision is taken afterward to map the estimated values to the closest modulated symbols, as such, $\tilde{\mathbf{s}}_{\lambda_{i}}$ is the solution to (7). The last iteration provides the data stream estimates for each detected node.

\section{PERFORMANCE METRICS}

\section{A. Detection evaluation}

1) Detection Error Rate: This metric is used for the first approach focusing on user detection only. The number of message arrivals follows a Poisson law with parameter $\mu \mathrm{m}$. $\mu=\mu_{0} N$ where $\mu_{0}$ is the message arrival rate per node. Let $X$ denote the random variable of message arrivals number and $\mathbb{P}_{m}(X=k)$ the probability of $k$ message arrivals in a slot of length $m$ symbols. According to the Poisson law:

$$
\mathbb{P}_{m}(X=k)=e^{-\mu m} \frac{(\mu m)^{k}}{k !}
$$

The Detection Error Rate (DER) depends on the expected messages error number, such as:

$D E R=\frac{\sum_{k=\kappa+1}^{\infty}\left(k \mathbb{P}_{m}(X=k)\right)}{\sum_{k=0}^{\infty}\left(k \mathbb{P}_{m}(X=k)\right)}=\frac{\sum_{k=\kappa+1}^{\infty}\left(k \mathbb{P}_{m}(X=k)\right)}{\mathbb{E}[X]}$

where $\mathbb{E}[X]$ is known to be equal to the parameter $\mu \mathrm{m}$.

2) Detection Success Rate: In the second approach, the detection efficiency is quantized by the Detection Success Rate (DSR). Let $\lambda_{\mathcal{N}_{a}}$ denote the detected set,

$$
D S R=\frac{\operatorname{card}\left(\lambda_{\mathcal{N}_{a}}\right)}{N_{a}}=\frac{\operatorname{card}\left(\lambda_{K} \cap \mathcal{N}_{a}\right)}{N_{a}}
$$

3) Missed Detection and False Alarm: In order to have more details about the detection performance, we use two additional metrics: the Missed Detection (MD) and False Alarm (FA) ratios. Let $\lambda_{\overline{\mathcal{N}_{a}}}$ denote the FA (also known as the False Positive) set, MD and FA ratios are thus:

$$
\begin{gathered}
M D=\frac{\operatorname{card}\left(\mathcal{N}_{a} \cap \overline{\lambda_{\mathcal{N}_{a}}}\right)}{N_{a}}=1-D S R \\
F A=\frac{\operatorname{card}\left(\lambda_{\overline{\mathcal{N}_{a}}}\right)}{\operatorname{card}\left(\mathcal{N} \cap \overline{\mathcal{N}_{a}}\right)}=\frac{K-\operatorname{card}\left(\lambda_{\mathcal{N}_{a}}\right)}{N-N_{a}}
\end{gathered}
$$

\section{B. Estimation evaluation}

1) Symbol Error Rate: The estimation performance is evaluated by means of the classic Symbol Error Rate (SER) metric. $E r r_{n}$ denotes the erroneously estimated symbols of node $n$, SER can be expressed by:

$$
S E R=\frac{d * \operatorname{card}\left(\lambda_{\overline{\mathcal{N}_{a}}}\right)+\sum_{n \in \lambda_{\mathcal{N}_{a}}} \operatorname{card}\left(\operatorname{Err}_{n}\right)}{d * N_{a}}
$$

\section{Latency evaluation}

1) Latency: The latency can be defined as the sum of the waiting time (the maximum channel use number that a node would have to wait before being allowed to send its message), the transmission time and the decoding time. However, the decoding time can be neglected since, in this work, we consider that the BS is not limited from computation and power resources viewpoints. Moreover, as the transmission time varies 
according to the distance between the node and the BS, we will only take the static part, i.e. the message duration, into account. As a result, the latency should be approximately twice the message duration. As it is directly proportional to the message length, the latency of both access scheme can therefore be evaluated by the message length $m$.

\section{Detection Performance}

We firstly focus on the detection performance evaluation. We consider an ideal scenario and a simple use case with no data transmission but where the nodes aim to notify of their activity. In this kind of application [9], the decoder only has to detect the active nodes. We simplify the model, assuming that the transmissions occur in a high SNR regime (noise is neglected). We also assume that both the transmitters and the receiver have one antenna and that the channel is not subject to fading. As such, only the presence of competing communications makes the transmissions succeed or fail, according to their number. Especially, it is assumed that an ALOHA-based transmission is correctly detected if no other communications occur within the same time slot, i.e. if no collision occurs. NOMA can handle a few simultaneous transmissions, according to the code length.

Theorem 6 of [14] defines the length of measurement vectors (in relation to the sparsity level $k$ of the signal, its dimension $N$ and some positive constants $\delta$ and $\beta$ ) as the necessary length for a successful reconstruction of the signal via OMP using a predefined measurement matrix, with a probability of success above $1-\delta$. The length $m$ is defined as $\beta k \log (N / \delta)$. Further, the authors observe for a success probability $P_{s}$ of $99 \%$, that $m$ can be approximated by $2 k \log (N)$. Moreover, [10] confirms this estimation by showing that $m=2 k \log (N-k)$ is sufficient for reconstruction under asymptotic conditions. The authors clarify that, when $k$ is unknown but between $k_{\min }$ and $k_{\max }$, having $m=2 k_{\max } \log \left(N-k_{\min }\right)$ measurements is sufficient. We are therefore using the similar formula for the Gaussian codes length with the assumption that $k_{\min }=0$, leading to $m=2 k_{\max } \log (N)$. We assume that this code length allows to detect up to $k_{\max }$ simultaneous transmissions with $99 \%$ of success, according to the numerical model from [14]. Let $\kappa$ denote the maximal detected message number in a time slot: $\kappa_{\text {aloha }}=1$ and $\kappa_{\text {noma }}=k_{\max }$.

Recalling equation 9, the Detection Error Rates are:

$$
\begin{aligned}
& D E R_{\text {aloha }}=\frac{\sum_{k=2}^{\infty}\left(k \mathbb{P}_{m}(X=k)\right)}{\mathbb{E}[X]}=1-e^{-\mu_{0} N L / M} \\
& D E R_{\text {noma }}=P_{s} \frac{\sum_{k=k_{\max }+1}^{\infty}\left(k \mathbb{P}_{m}(X=k)\right)}{\mathbb{E}[X]}+1-P_{s} \\
& =1-P_{s} \cdot e^{-2 \mu_{0} N . k_{\max } \log (N)} \frac{\sum_{k=0}^{k_{\max }}\left(k \frac{\left(2 \mu_{0} N \cdot k_{\max } \log (N)\right)^{k}}{k !}\right)}{2 \mu_{0} N \cdot k_{\max } \log (N)}
\end{aligned}
$$

The DER of the two access schemes are shown in Fig. 1 for a range of node set size from $1.10^{3}$ to $25.10^{3}$. The results are given between 1 and $10^{-2}$ due to the upper bound approximation. The Poisson law parameter $\mu_{0}$ is set to $1 / 3.6 .10^{5}$ message

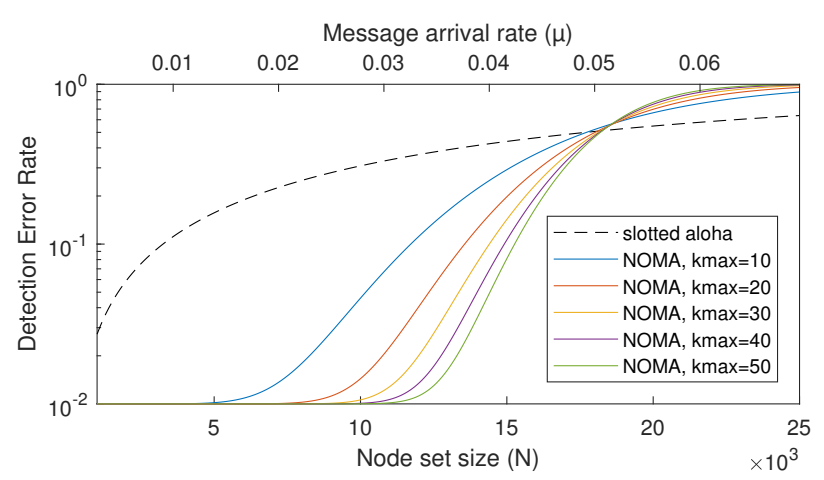

Fig. 1. Detection Error Rate of NOMA and ALOHA systems according to the node set size $N$

per symbol time, which corresponds to a mean activity of one message per node per hour with a modulation rate of $100 \mathrm{bps}$. The NOMA DER are bounded by the success probability threshold $P_{s}$, related to the approximation of the sufficient length from the observations of Tropp et al. and the curves might be shaped by this $10^{-2}$ bound for low node set size or message arrival rate. Nevertheless, we can observe that NOMA outperforms the slotted ALOHA system up to $N \approx 17.10^{3}$. For larger $N$, the message length of NOMA becomes too large to maintain a number of simultaneous active nodes below $k_{\max }$ in a time slot with a high probability, thus the fast degradation up to the point where the ALOHA system takes advantage of shortest time slots even if only one active node is allowed for a successful detection.

Fig.1 shows the benefit of NOMA with Gaussian codes compared to a slotted ALOHA for a range of large $N$. But this analysis suffers from several weak points: the model is dedicated to a network-level performance evaluation, showing the effect of collisions on both access techniques. However, it does not consider channel distortions, neither fading nor noise. Secondly, it also relies on approximations. The real performance of decoding algorithms are not exploited. Furthermore, this analysis is not adapted to data transmission evaluation. Therefore, a more sophisticated model, taking physical layer aspects into account, is presented in the next section.

\section{Detection and Estimation Performance}

In order to evaluate node detection probability and data estimation error rates, we implemented code domain NOMA and ALOHA-based systems with simulations under the more realistic transmission scenario described in section II-A.

a) Comparison of NOMA to ALOHA transmissions performance: Fig. 2 and 3 represent the detection and estimation performance of the confronted systems. The results are drawn as function of the SNR in the range $[-4 ; 20] \mathrm{dB}$, for different active nodes numbers (from $N_{a}=8$ to 30 ). The nodes set size is fixed to 80 , and the packets are all made of $d=100$ QPSK symbols. NOMA messages length $m$ is 500 symbols, while AlOHA messages are transmitted over 104 symbol time (or channel use). To keep a quasi-equivalent channel occupancy, 


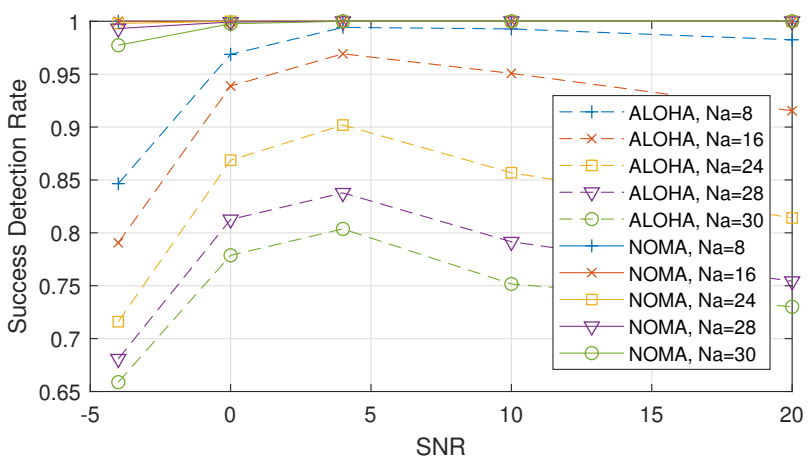

Fig. 2. AlOHA and NOMA Detection Success Rate

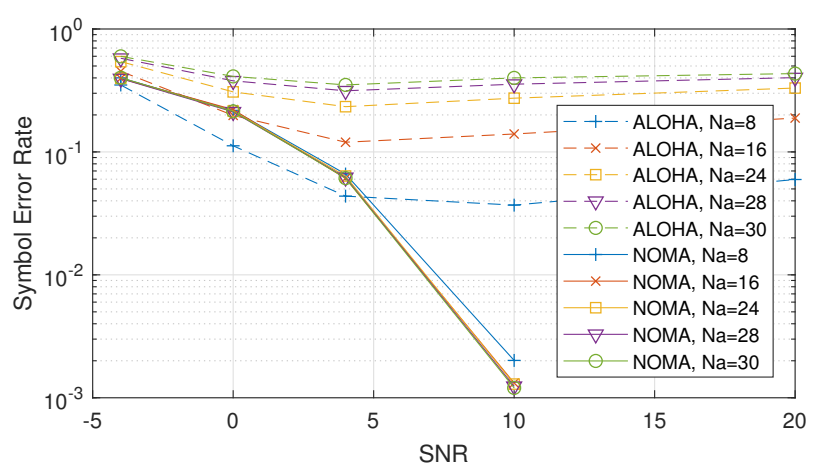

Fig. 3. AlOHA and NOMA Symbol Error Rate

the active nodes in AlOHA are uniformly associated to one of the 5 available time slots of the observation frame (as $\lceil 500 / 104\rceil=5$, the equivalent frame lasts 520 channel use). The BS possesses $\alpha=8$ antennas and runs $K=30$ iterations of either the SIC-based decoder for the Aloha system or the NBOMP decoder for the NOMA transmissions.

Fig. 2 shows that the DSR of NOMA is subject to little variations w.r.t. $N_{a}$ and to the SNR while the detection performance of ALOHA degrades when $N_{a}$ rises, and shows a local maximum when $S N R \approx 4 \mathrm{~dB}$. For the whole range of represented SNR and active nodes number, NOMA outperforms ALOHA in terms of DSR. Similarly, in Fig. 3, the performance divergence of ALOHA and NOMA can be observed. Indeed, the SER of NOMA remains quasi-insensitive to the active node number whereas the impact of the worse detection with ALOHA is clear. For SNR in $[-4,4] \mathrm{dB}$, we take for granted that, for both access schemes, when the SNR increases, the SER decreases. For SNR values above $4 \mathrm{~dB}$, the degradation of the SER of ALOHA is related to the decreasing DSR whereas the SER of NOMA continues to improve beyond $10^{-2}$. Even though the NOMA performance are better than the ALOHA as a general rule, one can nevertheless notice the superiority of the data estimation of the AlOHA system when $N_{a}=8$ for the lowest SNR values, although the detection probability of ALOHA is lower than that of NOMA. For larger $N_{a}$, NOMA takes advantage of the redundancy introduced in the coding scheme despites of the collisions. However, if the Missed Detections are not taken into account, i.e. when the SER is restricted to the active nodes that have been detected, the SER of NOMA and the worse SER of ALOHA even coincide for the range of lowest SNR values. In this case, NOMA also outperforms Aloha for any active nodes number as soon as SNR $>4 \mathrm{~dB}$.

Despites of the inter-dependence of the detection and the estimation tasks of the NBOMP or SIC-based algorithms, one can not state on a better efficiency of an access scheme compared to the other based on only one of the previous evaluation metrics. Indeed, the behaviors of the transmission techniques on the detection side don't directly predict the performance on the estimation side therefore the two metrics must be considered as a whole. Moreover, the choice of parameters such as $m$ or $K$ can be beneficial to a particular metric while degrading the results of another performance metric, as pointed out in the following paragraphs.

b) Latency versus SER: Let consider that a constant number of active nodes has access to the channel within a frame length, or channel use number, in order to transmit the same amount of data. In Fig. 4, $N a=8$ nodes among $N=80$ transmit $d=100$ data symbols. The messages with NOMA occupy the full frame whereas for ALOHA, the nodes keep the same time slot length (i.e. $m=d+\lceil L / M\rceil$ ) but their message arrivals number in a slot decreases with the increase of the available channel use number.

The results of the SER w.r.t. the available frame length are shown at the $16^{\text {th }}$ and the $30^{\text {th }}$ iteration, with $\alpha=8$, $S N R=10 d B$. Fig. 4 shows how NOMA SER drops sharply around 200 or 300 channel use (resp. for 16 and 30 iterations) whereas the ALOHA SER decreases slowly with the lengthening of the frame. This illustrates how latency trades with reliability for each access scheme: a longer $m$ in NOMA, allowing more redundancy, improves the estimation capability at the cost of an increase of the latency of the same order. On the opposite, the AlOHA system benefits from a short latency, independent of the frame length and allowing fewer concurrent accesses during a transmission, however not enough to compensate for the lower performance, dropped down by a low DSR. Another difference stems from the SER of NOMA that significantly

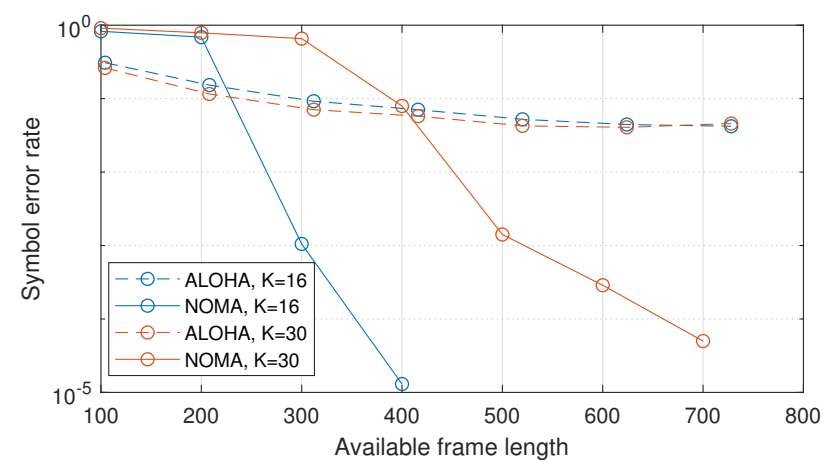

Fig. 4. SER versus available channel use frame length 
degrades by an iteration number increase while ALOHA is subject to an almost negligible estimation improvement. Indeed, at $N_{a}=8$, additional NBOMP iterations introduce FA. Since the estimation of the $K$ node signals is performed jointly, the estimated signals of active nodes are corrupted by the FA. The vertical gap between red and blue curves of NOMA is representative of this SER degradation. On ALOHA side, the slight difference is due to the loss in the DSR. Additional iterations allow to increase the detection probability thus the SER also improves.

c) Detection Error Trade-off: Fig. 5 represents the Detection Error Trade-off (DET) of NBOMP and ALOHA SIC-based decoder. The MD ratio is represented as a function of the FA rate for $N=80, N_{a}=24, \alpha=8, S N R=-10 d B, d=100$, $m_{\text {NOMA }}=500, m_{A L O H A}=104$ and $K$ varying between 10 and 40 iterations. One can make two observations: firstly, again NOMA has better MD and FA ratios than ALOHA for the same set of parameters. Moreover, one can note the efficient decrease of MD for NBOMP with a few iterations, but once $K$ reaches $N_{a}$ this tendency is inversed and the detection of a few more active nodes costs much more False Alarms. This makes it clear that the choice of the iterations number for the decoding algorithm also has a great impact on the detection performance.

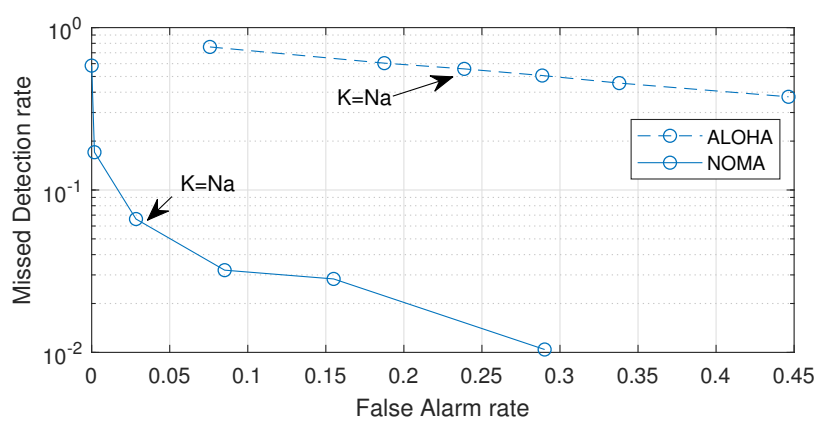

Fig. 5. MD vs FA ratios

\section{CONCLUSION}

The simulation studies performed in this paper highlight the performance gain that can be achieved thanks to a code domain NOMA random access. Despite longer packets resulting in a higher channel occupancy rate, the NOMA outperforms a classical ALOHA scheme, thanks to a robust multi-user decoder technique. Our future work will consist in optimizing the coding scheme to maximize the system performance, which may be measured by the nodes density that is achievable for a fixed bandwidth and under some error rate constraint.

It is worth mentioning that the multi-user receiver used in this paper implements a multi-user LS receiver which suffers from a high complexity. Even if the algorithmic complexity at the BS can be considered negligible compared to the nodes complexity, this technique cannot directly scales for very dense networks with hundreds and even thousands nodes. Sub-optimal algorithms will be necessary.

\section{REFERENCES}

[1] X. Xiong, K. Zheng, R. Xu, W. Xiang, and P. Chatzimisios, "Low power wide area machine-to-machine networks: Key techniques and prototype," IEEE Communications Magazine, vol. 53, no. 9, pp. 64-71, 2015.

[2] P. Schulz, M. Matthe, H. Klessig, M. Simsek, G. Fettweis, J. Ansari, S. A. Ashraf, B. Almeroth, J. Voigt, I. Riedel, A. Puschmann, A. Mitschele-Thiel, M. Muller, T. Elste, and M. Windisch, "Latency Critical IoT Applications in 5G: Perspective on the Design of Radio Interface and Network Architecture," IEEE Communications Magazine, vol. 55, no. 2, pp. 70-78, feb 2017. [Online]. Available: http://ieeexplore.ieee.org/document/7842415/

[3] N. Abramson, "THE ALOHA SYSTEM: another alternative for computer communications," in AFIPS '70 (Fall): Proceedings of the November 17-19, 1970, fall joint computer conference. New York, New York, USA: ACM Press, 1970, pp. 281-285. [Online]. Available: http://portal.acm.org/citation.cfm?doid=1478462.1478502

[4] S. Ghez, S. Verdu, and S. Schwartz, "Stability properties of slotted Aloha with multipacket reception capability," IEEE Transactions on Automatic Control, vol. 33, no. 7, pp. 640-649, jul 1988. [Online]. Available: http://ieeexplore.ieee.org/document/1272/

[5] M. Shirvanimoghaddam, M. Dohler, and S. J. Johnson, "Massive NonOrthogonal Multiple Access for Cellular IoT: Potentials and Limitations," IEEE Communications Magazine, 2017.

[6] E. J. Candès, J. Romberg, and T. Tao, "Robust uncertainty principles: Exact signal reconstruction from highly incomplete frequency information," IEEE Transactions on Information Theory, 2006.

[7] S. Foucart and H. Rauhut, A Mathematical Introduction to Compressive Sensing, 1st ed. Birkhäuser, New York, NY, 2013. [Online]. Available: http://link.springer.com/10.1007/978-0-8176-4948-7

[8] G. Wunder, C. Stefanovic, P. Popovski, and L. Thiele, "Compressive coded random access for massive MTC traffic in 5G systems," in 2015 49th Asilomar Conference on Signals, Systems and Computers. IEEE, nov 2015, pp. 13-17. [Online]. Available: http://ieeexplore.ieee.org/document/7421050/

[9] A. K. Fletcher, S. Rangan, and V. K. Goyal, "On-off random access channels: A compressed sensing framework," CoRR, vol. abs/0903.1022, 2009. [Online]. Available: http://arxiv.org/abs/0903.1022

[10] A. Fletcher and S. Rangan, "Orthogonal matching pursuit from noisy measurements: A new analysis," Proc. Neural Inf. Processing Syst. 22, pp. 1-9, 2009. [Online]. Available: https://papers.nips.cc/paper/3784-orthogonal-matchingpursuit-from-noisy-random-measurements-a-new-analysis.pdf

[11] R. Xie, H. Yin, X. Chen, and Z. Wang, "Many Access for Small Packets Based on Precoding and Sparsity-Aware Recovery," IEEE Transactions on Communications, vol. 64, no. 11, pp. 4680-4694, nov 2016. [Online]. Available: http://ieeexplore.ieee.org/document/7558184/

[12] H. Nikopour and H. Baligh, "Sparse code multiple access," in IEEE International Symposium on Personal, Indoor and Mobile Radio Communications, PIMRC, 2013.

[13] J. Choi, "NOMA-Based Random Access With Multichannel ALOHA," IEEE Journal on Selected Areas in Communications, vol. 35, no. 12, pp. 2736-2743, dec 2017. [Online]. Available: http://ieeexplore.ieee.org/document/8085106/

[14] J. A. Tropp and A. C. Gilbert, "Signal recovery from random measurements via orthogonal matching pursuit," IEEE Transactions on Information Theory, vol. 53, no. 12, pp. 4655-4666, 2007. 